\title{
Distribution and characteristics of amphibole asbestos fibres, measured with the light microscope, in the left lung of an insulation worker
}

\author{
A MORGAN AND A HOLMES \\ From the Environmental and Medical Sciences Division, Atomic Energy Research Establishment, Harwell, \\ Oxfordshire OX11 ORA, UK
}

ABSTRACT Concentrations of uncoated and coated amphibole fibres were measured postmortem in samples taken from the periphery of both upper and lower lobes of the left lung of an insulation worker. Similar measurements were made on a more limited range of samples from elsewhere in the lung and on a hilar lymph node. The mean concentration of uncoated fibres in the upper lobe was twice that in the lower. Significant differences were observed in the mean concentration of uncoated fibres in different regions of the periphery, the diaphragmatic region having the lowest value. Variations in the concentrations of both uncoated and coated fibres in the costal region of the lower lobe may have been related to the effect of rib structures on ventilation. Concentrations of fibrous and non-fibrous dust were well correlated in the peripheral samples. The length distributions of uncoated and coated fibres were also measured and differences detected in fibres from the various regions of the lung. This work emphasises the care required in sampling lung tissue in order to obtain representative material for the determination of fibre concentration.

Although many workers have measured concentrations of asbestos fibres in samples of human lung postmortem, few studies have been performed of variations in the concentrations and characteristics of asbestos fibres within a single lung. Sebastien et al compared the concentrations of fibres in the central and peripheral regions of upper and lower lobes of lungs using both optical and transmission electron microscopy. For asbestos workers with heavy exposure, they found that fibre concentrations were greater in the upper lobe but the difference was not apparent in a second group with less exposure to asbestos. In the latter an accumulation of asbestos fibres, especially of chrysotile, was shown in peripheral areas. In a more recent paper Sebastien $e t$ $a l^{2}$ compared the concentrations of amphibole and chrysotile fibres in samples of tissue from the parietal pleura and parenchyma of a series of lung samples using transmission electron microscopy. This study showed that there was no correlation between the concentrations of fibres in the two regions but confirmed their earlier observation that the proportion of chrysotile fibres was greater at the periphery of the lung.

Received 9 November 1981

Accepted 18 February 1982
The objective of the present study was to carry out a more detailed analysis of asbestos fibres in a sagittal section of human lung taken postmortem. In particular, it was proposed to study variations in fibre concentration, in fibre length, and in asbestos body formation. The investigation was carried out with optical microscopy which, under the conditions used, can detect fibres with diameters down to $0.2 \mu \mathrm{m}$. Optical microscopy detects only about $10-15 \%$ of crocidolite fibres ${ }^{3}$ but a greater proportion of the intrinsically thicker amphiboles, amosite, and anthophyllite. Few, if any, chrysotile fibres are detected as those found in lung are virtually all under $0.2 \mu \mathrm{m}$ in diameter. ${ }^{4}$

\section{Materials and methods}

\section{CASE HISTORY}

The sagittal section of left lung used in the present study was obtained postmortem from a 57 year-old man who had been exposed to asbestos for 37 years as an insulation engineer. The cause of death was a poorly differentiated squamous cell carcinoma of the bronchus that had shown secondary spread to the pleura. There was no evidence of mesothelioma. Lung tissue showed macroscopic and microscopic evidence of fairly substantial asbestosis. 


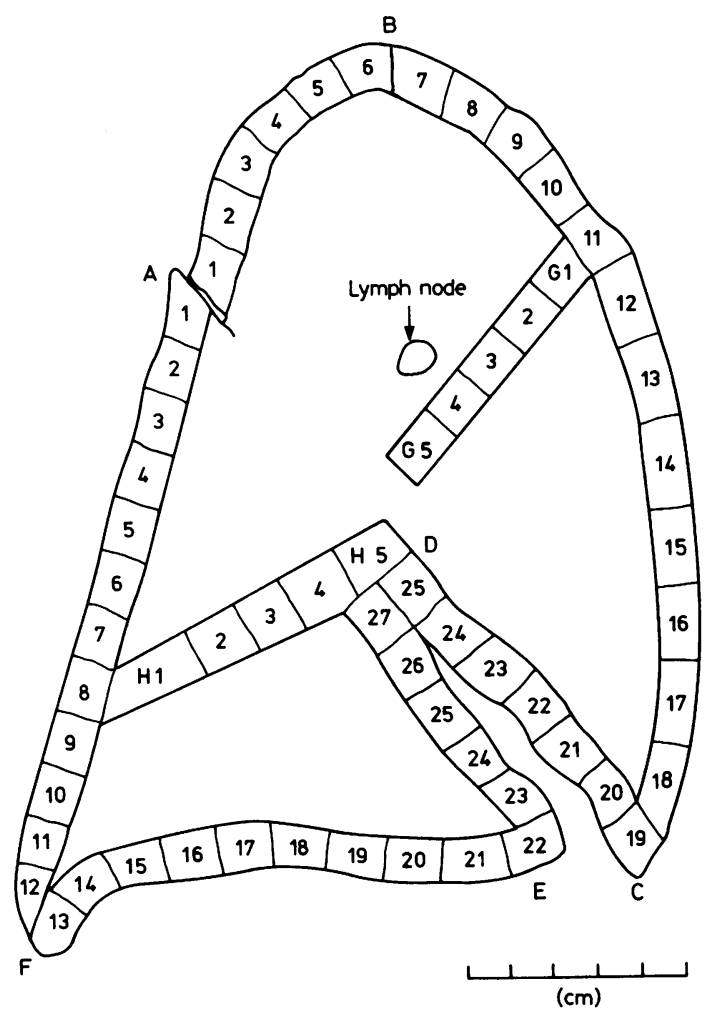

Fig 1 Diagram of sagittal slice of left lung showing from where samples were taken for analysis.

\section{SAMPLING OF LUNG}

A diagram of the sagittal slice (fig 1) indicates the position from which blocks were taken for analysis. Essentially, a $1 \mathrm{~cm}$ layer was taken from the periphery of both upper and lower lobes including the oblique fissure. Blocks, about $1.3 \mathrm{~cm}$ in length, were cut from this layer for analysis. In addition, transects were taken from both upper (G1-G5) and lower (H1-H5) lobes. Finally, a lymph node was excised from the hilar region of the upper lobe.

ANALYSIS OF LUNG TISSUE

From each block of tissue about $0.5 \mathrm{~g}$ was weighed accurately and digested with sodium hypochlorite solution (Parozone, Jeyes Ltd) as described by
Morgan and Holmes. 5 The remainder of the block was also weighed and then dried to constant weight at $110^{\circ} \mathrm{C}$ to obtain the wet/dry weight ratio. After digestion was complete, lipids were extracted with diethyl ether and an appropriate aliquot of the resulting suspension filtered through a $2.5 \mathrm{~cm}$ diameter Millipore GS membrane filter (pore size $0.22 \mu \mathrm{m}$ ). The filter was washed well with water and allowed to dry. Half the filter was placed on a microscope slide and cleared with a dimethylformamide/acetic acid/ water mixture. ${ }^{12}$ The other half was retained for examination with the electron microscope.

Uncoated rectilinear fibres greater than $2.5 \mu \mathrm{m}$ in length were counted and sized under phase contrast with a Porton-type eyepiece graticule at a magnification of $\times 640$ (Zeiss Photomicroscope III). Coated fibres were counted and sized at a magnification of $\times 256$, although a higher magnification was used in doubtful cases. All fibres showing any evidence of coating were classified as "coated" even though they may have been uncoated for most of their length. Wherever possible, the lengths of at least 200 uncoated and of $\mathbf{1 0 0}$ coated fibres were measured and classified in the following categories: $2 \cdot 5-4 \cdot 9,5-9 \cdot 9$, $10-19.9,20-39.9,40-79.9$ and $>80 \mu \mathrm{m}$. From the numbers in the various categories count median lengths (CMLs) were determined.

To establish the precision of the technique, six equal aliquots of lung digests were filtered and the concentrations and CMLs of both uncoated and coated fibres measured. The results are given in table 1 . In all but one case the coefficient of variation was about $10 \%$ or less.

To determine the proportion of amphibole fibres detected with the optical microscope, three samples from different regions of the periphery of the lung were also examined with the electron microscope at a magnification of $\times 1760$. Randomly selected areas were photographed and the prints used for fibre counting and measurement of count median diameters (CMDs) and CMLs. Categorised data were used for this purpose.

There was quite a high concentration of nonfibrous dust in the lungs of the subject. Concentrations of this were estimated on an arbitrary scale of one to six using the same slides as were used for the assessment of fibre concentrations.

Table 1 Results of six replicate measurements of fibre concentrations and length made on equal aliquots of fibre suspensions prepared from two samples of lung tissue. (Mean $\pm S D$ )

\begin{tabular}{|c|c|c|c|c|}
\hline \multirow[t]{2}{*}{ Sample } & \multicolumn{2}{|c|}{ Fibre concentration $\left(\times 10^{6} / g\right.$ dry wt) } & \multicolumn{2}{|l|}{$C M L(\mu m)$} \\
\hline & Uncoated & Coated & Uncoated & Coated \\
\hline $\begin{array}{l}\text { Upper lobe } 11 \\
\text { Lower lobe } 17\end{array}$ & $\begin{array}{r}50 \cdot 0 \pm 2.9 \\
7.0 \pm 0.8\end{array}$ & $\begin{array}{l}5.57 \pm 0.85 \\
0.93 \pm 0.08\end{array}$ & $\begin{array}{l}6 \cdot 6 \pm 0 \cdot 6 \\
9 \cdot 8 \pm 0 \cdot 2\end{array}$ & $\begin{array}{l}23 \cdot 3 \pm 1 \cdot 7 \\
29 \cdot 7 \pm 2 \cdot 7\end{array}$ \\
\hline
\end{tabular}




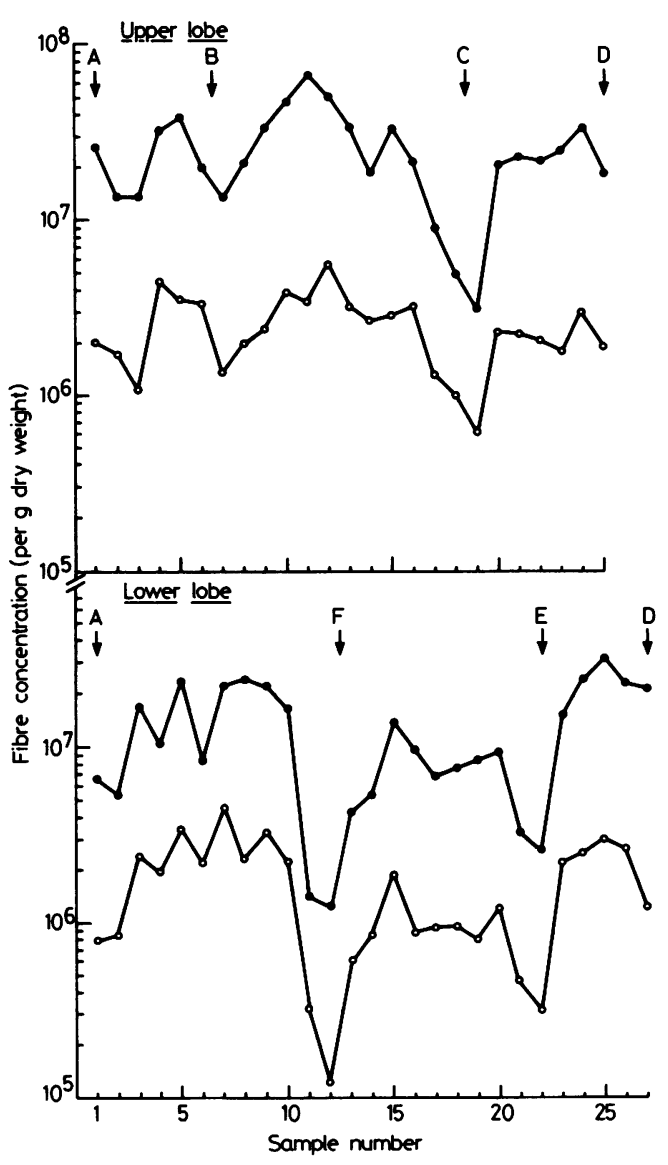

Fig 2 Concentrations of uncoated ( $\bullet$ ) and coated (o) amphibole fibres in peripheral samples from upper and lower lobes.

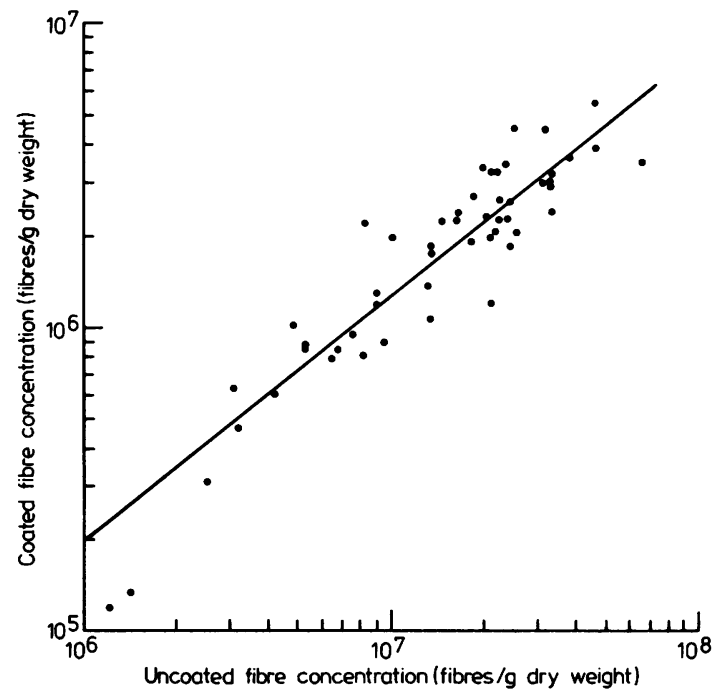

Fig 3 Correlation between concentrations of uncoated and coated amphibole fibres in peripheral samples from upper and lower lobes $(r=0.93 ; p<0.001)$.

\section{Results}

The results of measuring the concentrations of uncoated and coated fibres in the peripheral samples from the upper and lower lobes are shown in fig 2 . To facilitate cross reference, the extremities of each lobe, indicated by the letters shown in fig 1 , are included in fig 2 at the appropriate points. It is immediately apparent that there was a close correlation between the concentration of uncoated and coated fibres. This correlation, in $\log \log$ form, is shown in fig 3 and is significant $(r=0.93, p<0.001)$.

Table 2 Summary of measurements of concentrations and count median lengths of uncoated and coated fibres in upper and lower lobes. (Mean $\pm S E M)$

\begin{tabular}{|c|c|c|c|c|c|c|}
\hline \multirow[t]{2}{*}{ Location } & \multirow[t]{2}{*}{ Blocks } & \multicolumn{3}{|c|}{ Fibre concentration $\left(\times 10^{6} / \mathrm{g} d r y w t\right)$} & \multicolumn{2}{|l|}{$C M L(\mu m)$} \\
\hline & & Uncoated & Coated & $\%$ Coated & Uncoated & Coated \\
\hline $\begin{array}{l}\text { Upper lobe } \\
\text { A-B } \\
\text { B-C } \\
\text { C-D } \\
\text { G }\end{array}$ & $\begin{array}{r}1-6 \\
7-19 \\
20-25 \\
1-5\end{array}$ & $\begin{array}{l}24 \cdot 0 \pm 4 \cdot 1 \\
29 \cdot 3 \pm 5 \cdot 2 \\
20 \cdot 7 \pm 3 \cdot 4 \\
34 \cdot 6 \pm 5 \cdot 8\end{array}$ & $\begin{array}{l}2 \cdot 7 \pm 0 \cdot 5 \\
2 \cdot 7 \pm 0 \cdot 4 \\
2 \cdot(0 \pm 0 \cdot 3 \\
2 \cdot 2 \pm 0 \cdot 4\end{array}$ & $\begin{array}{r}10 \cdot 1 \pm 1.2 \\
9.9 \pm 1.0 \\
9.8 \pm 1.2 \\
5.9 \pm 0.5\end{array}$ & $\begin{array}{r}9 \cdot 2 \pm 0 \cdot 3 \\
7 \cdot 3 \pm 0 \cdot 1 \\
10 \cdot 0 \pm 0 \cdot 3 \\
7 \cdot 7 \pm 0 \cdot 7\end{array}$ & $\begin{array}{l}27.2 \pm 0.5 \\
25.8 \pm 0.7 \\
32.9 \pm 0.6 \\
33.2 \pm 1.1\end{array}$ \\
\hline Overall mean & & $27 \cdot 1 \pm 2 \cdot 6$ & $2 \cdot 5 \pm 0 \cdot 2$ & $9 \cdot 3 \pm 0 \cdot 6$ & $8 \cdot 4 \pm 0 \cdot 3$ & $28 \cdot 9 \pm 0 \cdot 7$ \\
\hline $\begin{array}{l}\text { Lower lobe } \\
\text { A-F } \\
\text { F-E } \\
\text { E-D } \\
H^{*}\end{array}$ & $\begin{array}{r}1-12 \\
13-22 \\
23-27 \\
1-5\end{array}$ & $\begin{array}{r}1.3 \cdot 2 \pm 2 \cdot 5 \\
7 \cdot 0 \pm 1 \cdot 1 \\
22 \cdot 9 \pm 2 \cdot 6 \\
28 \cdot 1 \pm 7 \cdot 6\end{array}$ & $\begin{array}{l}2 \cdot(0 \pm 0.4 \\
0.9 \pm 0 \cdot 1 \\
2 \cdot 3 \pm 0 \cdot 3 \\
1.9 \pm 0 \cdot 6\end{array}$ & $\begin{array}{r}13 \cdot 6 \pm 1 \cdot 1 \\
11 \cdot 3 \pm 0.5 \\
9.3 \pm 1.2 \\
6 \cdot 4 \pm 0.6\end{array}$ & $\begin{array}{r}9 \cdot 1 \pm 0.7 \\
11.2 \pm 0.8 \\
8.6 \pm 0.2 \\
7.3 \pm 0.2\end{array}$ & $\begin{array}{l}31 \cdot 1 \pm 1 \cdot 2 \\
31 \cdot(0 \pm 0.7 \\
33 \cdot 6 \pm 1 \cdot 9 \\
34 \cdot 5 \pm 2 \cdot 9\end{array}$ \\
\hline Overall mean & & $14 \cdot 7 \pm 1 \cdot 9$ & $1 \cdot 7 \pm 0 \pm 2$ & $11 \cdot 3 \pm 0 \cdot 7$ & $9 \cdot 4 \pm 0 \cdot 4$ & $32 \cdot 2 \pm 0 \cdot 7$ \\
\hline
\end{tabular}

${ }^{*}$ Excluding $\mathrm{H} 3$. 
It may be seen that, on average, there were about 10 times as many optically visible uncoated fibres as coated in tissue taken at the periphery of the lung.

It is also apparent from fig 2 that the concentration of both uncoated and coated fibres fell quite sharply at the extremities of each lobe. Between the extremities there was generally a rather rapid increase to a relatively constant concentration in regions closer to the hilum. An exception was the costal region of the lower lobe, where there appeared to be a well-defined pattern of alternating peaks and troughs in the concentrations of both uncoated and coated fibres.

In table 2 are given the mean values for the concentrations of uncoated and coated fibres in the various regions of the periphery of the lung that are defined in the first column. Mean values for the transects are also included. Sample H3 was excluded as it was composed mainly of large conducting airways and the fibre concentration was low. For both the upper and lower lobes mean concentrations of uncoated fibres were greatest in the central transects but the same did not apply to coated fibres. The overall mean concentration of uncoated fibres in the upper lobe was almost twice that of the lower but the difference was less pronounced for coated fibres. Within each lobe there were significant differences in fibre concentration beteen peripheral regions. In the lower lobe, for example, the mean uncoated fibre concentration in the costal pleura was significantly $(p<0.05)$ greater than in the diaphragmatic pleura. Differences were less pronounced in the upper lobe.

As shown in table 2, CMLs of optically visible fibres in the upper and lower lobes averaged 8.4 and $9 \cdot 4 \mu \mathrm{m}$ respectively. These values are slightly less than those reported for the British gas-mask factory workers exposed to crocidolite, which generally fell in the $10-15 \mu \mathrm{m}$ range. ${ }^{3}$ The greatest CMLs for uncoated fibres were found in the diaphragmatic region where the mean value was $11 \cdot 2 \mu \mathrm{m}$. The CMLs of uncoated fibres in the central transects were lower, possibly due to the involvement of more lymphatic tissue. The CML of uncoated fibres in the lymph node excised from the upper lobe was only $3.4 \mu \mathrm{m}$. This was considerably lower than for any other sample and may be accounted for by the more ready transfer of short fibres to the pulmonary lymphatics.
The mean concentrations of coated fibres were less variable than for uncoated but, as with uncoated fibres, levels were higher in the upper lobe. In the upper and lower lobes $9.3 \%$ and $11.3 \%$ of the fibres present were coated respectively. These values are quite close to the mean of $11 \%$ reported by Morgan and Holmes $^{3}$ for 22 gas-mask factory workers exposed to crocidolite. As may be seen from table 2, however, there were variations within lobes, central samples having significantly $(\mathrm{p}<0.01)$ lower values on average than peripheral.

The mean CMLs of coated fibres in the upper and lower lobes were 29 and $32 \mu \mathrm{m}$ respectively. The individual values fell in the same range as those reported for gas-mask factory workers. ${ }^{3}$ Few of the coated fibres were distorted or had clubbed ends, features which appear to be associated with considerably greater fibre concentrations than those encountered in the present case.

The results of measurements of CMDs and CMLs of fibres with the electron microscope are given in table 3. Corresponding values of CMLs measured by optical microscopy are included, together with an estimate of the percentage of all fibres detected with electronmicroscopy that were also detected with optical microscopy.

\section{Discussion}

The amphibole fibres in the lungs of the subject investigated were not identified. Nevertheless, the fact that the CMDs were greater than those observed in gas-mask factory workers exposed to crocidolite ${ }^{3}$ indicates that exposure to amosite may have occurred. In cases exposed to crocidolite only $10-15 \%$ of fibres are detected with the light microscope whereas in the present case, as shown in table 3, the proportion was nearer $50 \%$.

CMLs measured with the optical microscope were on average about $25 \%$ greater than when measured with the electron microscope. This discrepancy is due partly to the difficulty in detecting fibres less than 2 $\mu \mathrm{m}$ in length with the former and also to the fact that. as shown by Pooley and Clarke, ${ }^{+}$the diameter of crocidolite and amosite fibres increases with their length so that longer fibres are detected more readily.

From the results obtained, it is clear that the mean

Table 3 Comparison of measurements with the electron and optical microscopes

\begin{tabular}{|c|c|c|c|c|}
\hline \multirow[t]{2}{*}{ Sample } & \multicolumn{2}{|c|}{ Electron microscope } & \multicolumn{2}{|c|}{ Optical microscope } \\
\hline & $C M D(\sigma g)$ & $C M L(\sigma g)$ & $C M L(\sigma g)$ & $\begin{array}{l}\% \text { fibres } \\
\text { detected }\end{array}$ \\
\hline $\begin{array}{l}\text { Upper lobe } 11 \\
\text { Lower lobe } 5 \\
\text { Lower lobe } 15\end{array}$ & $\begin{array}{l}(0.19(2.7) \\
(0.16(2.3) \\
(0.11(2.1)\end{array}$ & $\begin{array}{l}5 \cdot 6(2 \cdot 1) \\
7 \cdot 6(2 \cdot 4) \\
8 \cdot 1(3 \cdot 5)\end{array}$ & $\begin{array}{r}7 \cdot()(2 \cdot 1) \\
7 \cdot 9(2 \cdot 2) \\
11 \cdot 5(2 \cdot 2)\end{array}$ & $\begin{array}{l}68 \\
50 \\
40\end{array}$ \\
\hline
\end{tabular}


concentration of optically visible fibres was greater in the upper lobe than in the lower and that the lowest concentrations were found in the diaphragmatic pleura. This is consistent with observations of the pattern of deposition of fibrous ${ }^{6}$ and non-fibrous ${ }^{7}$ material in the respiratory tract of rodents in which it has been shown that the apical lobe of the right lung has a higher concentration of particules than the mean and the lower lobes a lower. This effect is attributed to differences in the morphology of the conducting airways associated with the various lobes and the disproportion increases with aerodynamic diameter. ${ }^{78}$ The fact that, as shown in table 3 , fibres in the upper lobe appear to have a greater CMD than those in the lower may also be associated with the same factors. Sebastien $e t a^{1}$ also found higher fibre concentrations in the upper lobes of asbestos workers with heavy exposure. Lung fibrosis was present in all their cases, and it was suggested that the predominance of fibrosis in the lower lobes could influence retention in this area.

The pattern of fibre concentrations at the periphery of the lung indicates that it is determined primarily by ventilation. For example, the fibre concentrations at the extremities of each lobe are, relatively, much lower than elsewhere because these regions are poorly ventilated. The alternating pattern of peaks and troughs in concentration observed in the costal pleura may be related to the distribution of airways within the lung or to the effects of the rib cage on ventilation. Barnes, ${ }^{9}$ in a study of the distribution of inhaled radionuclides in the lungs of dogs and monkeys, showed, using autoradiography, that the particles were deposited in a striated pattern near the costal surface, apparently related to rib structures. Greatest concentrations were found below the ribs.

In both the upper and lower lobes the mean fibre concentrations were greatest in the central transects but the same did not apply to coated fibres. An increase in the concentration of fibres with distance from the pleura has been reported by Ophus et a $l^{\prime \prime}$ in studies using the scanning electron microscope.

To obtain some idea of whether the fibrous dust was distributed in a similar manner to the nonfibrous, levels of the latter were scored subjectively on a scale of one to six. In fig 4 the total (uncoated plus coated) fibre concentrations in the peripheral samples of the lung are plotted against the levels of non-fibrous dust. Clearly, there is a close correlation indicating that the same factors determined the distribution of non-fibrous dust and optically visible fibres. In central samples the levels of fibrous and nonfibrous dust were less well correlated.

The CMLs of uncoated and coated fibres are not independent variables. In general, long fibres are coated more readily than short and thick more readily

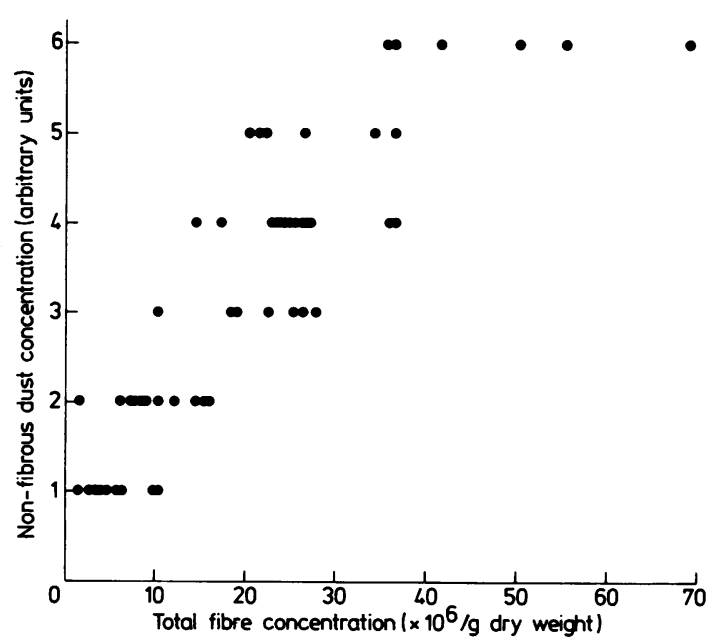

Fig. + Correlation between total fibre concentrations (uncoated and coated) and non-fibrous dust measured on an arbitrary scale of one to six.

than thin. ${ }^{11}$ If the longer fibres are coated more readily in one region of the lung than in another this will have the effect of reducing the CMLs of both uncoated and coated fibres. To study this aspect, the probability of fibres in the length range $20-40 \mu \mathrm{m}$ becoming coated was estimated for peripheral samples from the lower lobe. This range was selected

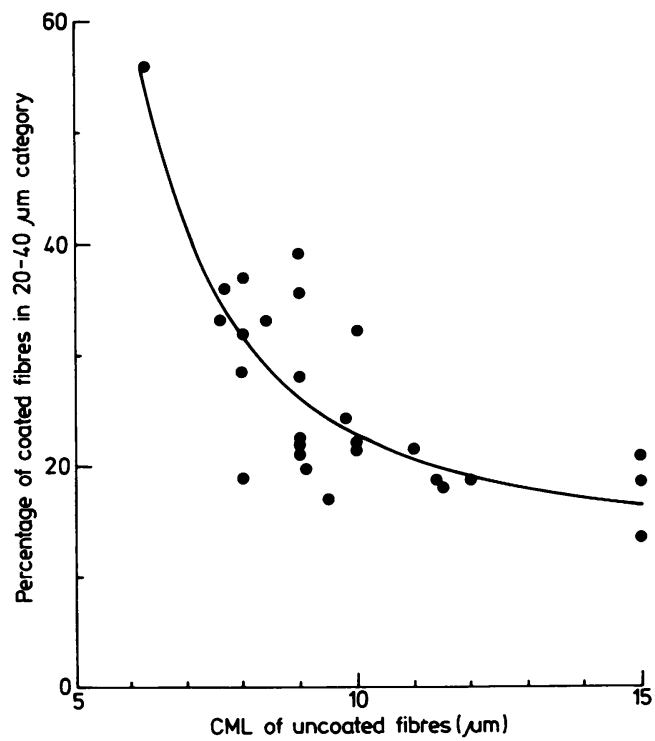

Fig 5 Correlation between count median length of uncoated fibres in samples from periphery of lower lobe and percentage of coated fibres in 20-40 $\mu \mathrm{m}$ length category. 
because a reasonable proportion of both uncoated and coated fibres fell within it so that the probability of coating could be estimated relatively accurately. Values from $14 \%$ to $56 \%$ were obtained with a mean of $26 \%$. This may be compared with values ranging from $6 \%$ to $34 \%$ for single samples obtained from the lungs of three gas-mask factory workers reported by Morgan. ${ }^{11}$ In fig 5 the results are plotted against the corresponding CMLs of uncoated fibres. As would be expected, the greater the probability of longer fibres being coated the lower the CML of uncoated fibres.

The main conclusion from this study is to emphasise the care required in sampling lung tissue to obtain representative material for analysis of fibre concentration. In this study the ratios between the highest and lowest concentrations of uncoated fibres were 21 and 26 for the upper and lower lobe respectively. Clearly, there is an advantage in taking samples from the periphery of the lung as the involvement of conducting airways is minimal, but the extremities should be avoided due to the relatively low and unrepresentative concentrations in these regions. It is recommended that samples should be taken from predetermined sites and analysed either individually or pooled in such a manner that a mean value may be derived from a single measurement.

The effect of fibre concentrations on lung pathology has not been discussed. It is known that the clearance of fibres from the lung by macrophagemediated processes is impaired when fibre concentrations are very high. Although asbestosis was present in the lungs examined, the unusually high proportion of short fibres, observed in cases of high exposure to crocidolite, ${ }^{3}$ was not apparent.

We gratefully acknowledge the support of the Asbestosis Research Council and of the EEC Environmental Research Programme (Project 27777-10 ENV UK). We also thank members of the Asbestosis Research Council for their constructive comments. The sample of lung was provided by $\mathrm{Dr}$ J S P Jones, City Hospital, Nottingham, and the electron microscopy was carried out by Mr M J Heard and Mr R D Wiffen.

\section{References}

' Sebastien P, Fondimar A. Bignon J, Monchaux G, Desbordes J, Bonnaud G. Topographic distribution of asbestos fibres in human lung in relation to occupational and non-occupational exposure. In: Walton WH, ed. Proceedings of 4th international symposium on inhaled particles, Edinburgh, 1975. Part 2. Oxford: Pergamon Press. 1977:435-44.

2 Sebastien P. Janson X. Gaudichet A, Hirsch A, Bignon J. Asbestos retention in human respiratory tissues: comparative measurements in lung parenchyma and in parietal pleura. In: Wagner JC, ed. Proceedings of symposium on biological effects of mineral fibres, Lyon 1979. Vol 1. Lyon: International Agency for Research on Cancer, 1980:237-46. (IARC Scientific Publications No 30.)

${ }^{3}$ Morgan A. Holmes A. Concentrations and characteristics of amphibole fibres in the lungs of workers exposed to crocidolite in the UK gas-mask factories and elsewhere during the second world war. BrJ Ind Med 1982;39:62-9.

${ }^{4}$ Pooley FD, Clark NJ. A comparison of fibre dimensions in chrysotile, crocidolite and amosite particles from samples of airborne dust and from post mortem lung tissue specimens. In: Wagner JC, ed. Proceedings of symposium on biological effects of mineral fibres, Lyon 1979. Vol 1. Lyon: International Agency for Research on Cancer, 1980:79-86. (IARC Scientific Publications No 30.)

${ }^{5}$ Morgan A, Holmes A. Concentrations and dimensions of coated and uncoated asbestos fibres in the human lung. Br J Ind Med 1980;37:25-32.

${ }^{\circ}$ Morgan A, Black A, Evans N, Holmes A, Pritchard JN. Deposition of sized glass fibres in the respiratory tract of the rat. Ann Occup Hyg 1980;23:353-66.

${ }^{7}$ Morgan A, Black A, Lambert BE, Moores SR, Pritchard JN, Walsh M. Alveolar deposition of sized particles of ${ }^{239} \mathrm{PuO}_{2}$ in the mouse. Harwell: Atomic Energy Authority 1981. (UKAEA unclassified report AERE-R 10074.)

${ }^{8}$ Raabe OG, Hsu-Chi Y, Newton GJ, Phalen RF, Valesquez DJ. Deposition of inhaled monodisperse aerosols in small rodents. In: Walton WH, ed. Proceedings of 4th international symposium on inhaled particles, Edinburgh, 1975. Part 1. Oxford: Pergamon Press. 1977:3-20.

${ }^{9}$ Barnes JE. Distribution of inhaled radionuclides in the respiratory tract. Health Phys 1971;21:227-32.

${ }^{10}$ Ophus EM, Mowe G, Osen KK, Gylseth B. Scanning electron microscopy and $x$-ray microanalysis of mineral deposits in lungs of a patient with pleural mesothelioma. $B r J$ Ind Med 1980;37:375-81.

"Morgan A. Effect of length on the clearance of fibres from the lung and on body formation. In: Wagner JC, ed. Proceedings of symposium on biological effects of mineral fibres, Lyon, 1979. Vol 1. Lyon: International Agency for Cancer Research, 1980:329-35. (IARC Scientific Publications No 30.)

12 Le Guen JMM, Galvin S. Clearing and mounting techniques for the evaluation of asbestos fibres by the membrane filter method. Ann Occup Hyg 1981;24:273-80. 